\title{
COMPORTAMENTO AMBIENTAL DO RATICIDA CUMATETRALILA EM PÓ DE CONTATO
}

\author{
SOLANGE PAPINI* \\ RITA DE CÁSSIA B. PRISCO** \\ LUIZ CARLOS LUCHINI ${ }^{* * *}$ \\ VERALÚCIAT. SAVOY****
}

\begin{abstract}
No controle químico de roedores na cidade de São Paulo é aplicado cumatetralila em pó de contato na entrada das tocas, ficando sujeito a ação das intempéries, podendo acarretar alterações no ingrediente ativo (i.a.) e transferência para outros ambientes. Para verificar essas possibilidades, o raticida foi aplicado em sistemas experimentais simulando as condições de campo. Amostras de solo e água foram coletadas semanalmente, durante 10 semanas, e analisadas por cromatografia a líquido de alta eficiência. Os dados obtidos foram utilizados para a avaliação da dissipação do i.a. e seu potencial de lixiviação no perfil do solo. Os resultados apontaram a presença de cumatetralila na camada de 0 a $5 \mathrm{~cm}$ do solo durante as 10 semanas. Detectou-se cerca de $75 \%$ do i.a. aplicado nas 3 primeiras semanas, em torno de $20 \%$ entre a $5^{\text {a }}$ e a $8^{\mathrm{a}}$ semanas e cerca de $7 \%$ na $10^{\mathrm{a}}$ semana. Nas amostras de água não foi detectado o i.a. durante as 10 semanas. Concluiu-se que o i.a. permanece no ambiente por pelo menos 10 semanas, sem atingir a água subterrânea considerando o tipo de solo utilizado.
\end{abstract}

PALAVRAS-CHAVE: HIDROXICUMARÍNICO; LIXIVIAÇÃO; PERSISTÊNCIA; RODENTICIDA.

* Bióloga, Doutora em Ecologia, Departamento de Controle da Qualidade Ambiental, Secretaria do Verde e Meio Ambiente, São Paulo, SP (e-mail: spapini@prefeitura.sp.gov.br).

** Engenheira Agrônoma, Pesquisador Científico, Laboratório de Química Ambiental, Instituto Biológico, São Paulo, SP (e-mail: rprisco@biologico.sp.gov.br).

*** Químico, Doutor em Química Ambiental, Laboratório de Ecologia de Agroquímicos, Instituto Biológico, São Paulo, SP (e-mail: luchini@biologico.sp.gov.br) .

**** Química, Pesquisador Científico, Laboratório de Química Ambiental, Instituto Biológico, São Paulo, SP (e-mail: savoy@biologico.sp.gov.br) . 


\section{INTRODUÇÃO}

O crescimento desordenado das grandes cidades permitiu o surgimento de moradias precárias em locais nem sempre adequados à construção civil (BRASIL, 2002). Além disso, os sistemas de saneamento, distribuição de água potável, tratamento de esgotos e coleta do lixo produzido tornaramse ineficientes para atender a demanda crescente (KOOKANA, DI e AYLMORE, 1996). Grande densidade populacional e saneamento básico deficiente em algumas cidades geraram condições propícias ao estabelecimento e desenvolvimento de animais sinantrópicos, muitos dos quais relacionados com diversas patogenias humanas (BRASIL, 2002; MORIN et al., 1989; VIEIRA, ALMEIDA e ALMEIDA, 1994). Entre esses animais, os ratos merecem destaque. As três espécies principais encontradas nas cidades brasileiras, Rattus norvegicus, Rattus rattus e Mus musculus, são euriécias. Adaptam-se facilmente às diferentes condições ambientais e apresentam alta taxa reprodutiva (BRASIL, 2002). Embora sejam espécies diferentes e tenham nichos ecológicos distintos estão relacionadas direta ou indiretamente com graves patogenias humanas. São transmissoras de leptospirose, causam infecções por meio de mordeduras e outros mecanismos e suas pulgas podem veicular a peste (ALMEIDA et al., 1994; COMER, PADDOCK e CHILDS, 2001; RAO et al., 2003; SILVA et al., 2003). Além do manejo ambiental, o controle desses roedores pode ser feito mediante hidroxicumarínicos nas formulações isca granulada, pó de contato e bloco parafinado (BRASIL, 2002). Os compostos raticidas são colocados no ambiente em que o animal se encontra para que ocorra a intoxicação mediante contato. $O$ cumatetralila formulado como pó de contato é aplicado, normalmente, nas entradas de tocas para que os animais ao entrarem em seus ninhos tenham o composto aderido ao pelo e dessa maneira ingiram o raticida ao se limparem (BRASIL, 2002).

O cumatetralila pode ser encontrado na forma de cristais e seu ponto de fusão está entre 172 e $176^{\circ} \mathrm{C}$. É solúvel em álcool e acetona, mas pouco solúvel em benzeno, tolueno, dietil éter e em dilalcali e na maioria dos solventes orgânicos (WORTHING e HANCE, 1991). O composto não é hidrolisado após 5 dias a $25^{\circ} \mathrm{C}$, decompõe-se rapidamente em soluções aquosas expostas a luz solar ou ultravioleta (UV) e apresenta meia-vida de 1 hora (TOXNET, 2004). Sua constante de dissociação (pKa) indica que o composto existe parcialmente na forma dissociada no ambiente e seus ânions geralmente não adsorvem fortemente ao carbono orgânico e argila (TOXNET, 2004). O fator de bioacumulação estimado (FBC) e seu coeficiente de partição octanol-água (Kow) indicam alto potencial de bioconcentração em organismos aquáticos (EPA, 2005). O coeficiente de partição carbono orgânico (Koc) sugere baixa mobilidade no solo (EPA, 2005) e pelo valor de sua pressão de vapor espera-se que não sofra volatilização a partir da superfície de solos seco e úmido (TOXNET, 2004).

O comportamento dos raticidas no ambiente depende de fatores como, por exemplo, as suas propriedades físicas e químicas, o tipo de formulação do ingrediente ativo (i.a.), a quantidade, freqüência e modo de sua aplicação, as características abióticas e bióticas do meio e as condições meteorológicas do local de aplicação (RACKE, 1990). De modo geral, quanto mais longa a permanência do composto no ambiente, mais acentuada será a contaminação ambiental (AMORIN et al., 2002). Os pesticidas podem ser transferidos por meio de arraste sobre a superfície do solo e da lixiviação, movimentandose nos sistemas aquáticos superficiais e subterrâneos (KOOKANA, DI e AYLMORE, 1996; PETROVIC et al., 1997; SEMA, 1993). Dessa maneira, podem provocar intoxicação e contaminação de organismos não-alvos (PAPINI et al., 2005).

Os raticidas atuam interferindo no processo de coagulação sangüínea, pois são compostos antagonistas estruturais da vitamina K. Além disso, exercem ação vasodestrutiva levando ao extravasamento sangüíneo (LARINI, 1999). Embora a ação dos raticidas hidroxicumarínicos no organismo seja bastante conhecida, existem poucas informações sobre o comportamento ambiental do i.a. cumatetralila formulado como pó de contato. Assim, procurou-se determinar sua persistência no ambiente sob condições urbanas de aplicação. Verificou-se a concentração do i.a. ou a presença de produtos de sua degradação após 10 semanas de exposição à intempéries e a possibilidade de contaminação do solo em que foi aplicado, bem como o potencial de contaminação dos recursos hídricos. 


\section{MATERIAL E MÉTODOS}

O padrão analítico cumatetralila certificado e com pureza de $99,9 \% \mathrm{~m} / \mathrm{m}$, do Laboratório de Química Ambiental (LQA) do Instituto Biológico (IB), foi utilizado no preparo de soluções-padrão com concentrações conhecidas para estabelecimento das condições de análise. O produto comercial na formulação pó de contato foi cedido pela Unidade de Vigilância Ambiental da Vila Mariana da Prefeitura do Município de São Paulo, e utilizado nos testes realizados para a extração do ativo da água e do solo.

\subsection{ESTABELECIMENTO DAS CONDIÇÕES DE ANÁLISE}

A detecção e quantificação do i.a. foram realizadas por cromatografia a líquido de alta eficiência (CLAE), com padronização externa, em cromatógrafo DIONEX, equipado com detetor ultravioletavisível (DIONEX UVD 170U/340U). A curva padrão do ativo foi estabelecida, em triplicata, a partir das soluções-padrão nas seguintes concentrações: 1,25; 2,50; 5,00 e 10,00 $\mu \mathrm{g}$ i.a mL-1 de acetonitrila:água (1:1 v/v), utilizando-se coluna fase não-polar RP-18 (250 x $46 \mathrm{~mm})$, comprimento de onda de $254 \mathrm{~nm}$, fase móvel metanol:solução a 1\% de $\mathrm{H}_{3} \mathrm{PO}_{4}$ na proporção de 75:25 (v/v) (MORIN et al., 1989), vazão de $1 \mathrm{~mL}$. $\mathrm{min}^{-1} \mathrm{e}$ temperatura de $30^{\circ} \mathrm{C}$. A partir dos resultados obtidos, com as diferentes concentrações de i.a., estabeleceu-se a curva padrão do ativo cumatetralila, com limites de detecção e de quantificação definidas pelo método de MEIER e ZÜND (1993).

\subsection{SOLO}

Coletou-se o solo utilizado nos experimentos em área não-exposta a agrotóxicos no Sítio Vila Viçosa, Município de Tuiuti, Estado de São Paulo. As amostras foram submetidas à análise físicoquímica no Laboratório de Análise de Solo e Planta do Instituto Agronômico. Amostras de solo foram analisadas quanto a capacidade de retenção de água (CMRA) (EMBRAPA, 2000). O solo utilizado nos ensaios foi reumedecido a $20 \%$ da CMRA.

\subsection{ESTUDO DE DISSIPAÇÃO E POTENCIAL DE LIXIVIAÇÃO - CUMATETRALILA}

Antes do início do experimento realizaram-se testes de recuperação do i.a. a partir de amostras de solo e de água tratadas com o produto formulado pó de contato. Amostras com $30 \mathrm{~g}$ de solo reumedecido a $20 \%$ da capacidade de campo foram tratadas com o raticida cumatetralila formulado como pó de contato $(4500 \mu \mathrm{g}$ i.a.) e colocadas em frasco com capacidade para $250 \mathrm{~mL}$. Foram acrescentados $50 \mathrm{~mL}$ de água destilada e efetuou-se a extração por agitação mecânica por 30 min. Em seguida foram adicionados $50 \mathrm{~mL}$ de acetonitrila, prosseguindo-se a agitação mecânica por mais $30 \mathrm{~min}$. Os extratos foram deixados para decantar por 24 horas e filtrados em papel filtro $(2,0 \mu \mathrm{m})$ umedecido em acetonitrila e, então submetidos à análise por CLAE. Amostras de 1,0 $\mathrm{mL}$ de água destilada foram tratadas com $1,0 \mathrm{~mL}$ de solução do padrão analítico cumatetralila com concentração conhecida e deixadas equilibrar por 30 minutos. Depois, foram acrescentados $10,0 \mathrm{~mL}$ de diclorometano em 3 extrações líquido-líquido de 5 minutos cada (muito lentamente deixando escapar o vapor). As fases foram separadas, todo o diclorometano foi coletado, concentrado à secura em aparelho rotavapor Büchi (modelo R111e) e ressuspendido em $5 \mathrm{~mL}$ de acetonitrila:água (1:1 v/v) para a análise por CLAE (detecção e quantificação do i.a).

Em área externa apropriada do Instituto Biológico (SP), sujeita a intempéries, foram montados os sistemas, simulando as condições de aplicação. $O$ ambiente experimental foi isolado com tela para evitar a contaminação de animais e colocado aviso de risco químico para evitar que pessoas estranhas entrassem no local.

Para o estudo de dissipação foram construídos mini-lisímetros, constituídos por tubo de policloreto de vinila (PVC) com $20 \mathrm{~cm}$ de diâmetro, cortado em pedaços com $25 \mathrm{~cm}$ de altura. No 
fundo de cada tudo colocou-se tampa de PVC acrescida de borracha isolante. Cada tubo foi furado a aproximadamente $5 \mathrm{~cm}$ do fundo, no qual colocou-se um pedaço de mangueira em poliuretano de $2,0 \mathrm{~mm} \times 0,5$ polegadas e $50 \mathrm{~cm}$ de comprimento.

Os mini-lisímetros foram montados da seguinte forma: 1) distribuição de pedregulhos no fundo formando camada de aproximadamente $3 \mathrm{~cm}$; 2) colocação de prato plástico para vaso de plantas furado sobre os pedregulhos; 3 ) instalação de tela sobre o prato plástico para vaso de modo a evitar que o solo se misturasse à água percolada; e 4) preenchimento com solo não-contaminado, deformado, até $20 \mathrm{~cm}$. Os tubos foram montados em sistema suspenso para que a água proveniente da chuva e percolada pelo perfil da coluna de solo fosse recolhida em garrafão de vidro com capacidade para 4 litros. Nos 30 mini-lisímetros montados foram aplicados $20 \mathrm{~g}$ de pó de contato sobre a superfície do solo. Os mini-lisímetros foram numerados de 1 a 30 , dispostos aleatoriamente sobre os estrados e cobertos com tela galinheiro. A mangueira de poliuretano com $50 \mathrm{~cm}$ de comprimento que saia de cada mini-lisímetro foi acoplada a um garrafão (também numerado de 1 a 30) para coleta de água correspondente.

As condições ambientais, pluviosidade e temperatura, foram monitoradas por meio de pluviômetro e termômetro de máxima e mínima, dispostos no local do experimento. A medição da pluviosidade ocorreu uma vez por semana, sendo as temperaturas mínima e máxima verificadas diariamente no local do experimento, exceto nos fins de semana e feriados.

Antes do início do experimento realizou-se a extração de amostras de solo tratado, imediatamente após aplicação do produto $\left(T_{\text {zero }}\right)$ para se confirmar os resultados obtidos nos testes de recuperação em escala real de trabalho. Para isso foram montados 2 mini-lisímetros como descrito acima, sendo aplicados $20 \mathrm{~g}$ de raticida sobre a superfície do solo. Após 1 hora, os sistemas foram desmontados e a primeira camada de solo $(0-5 \mathrm{~cm})$ retirada e pesada, determinando-se seu teor de umidade. Após homogeneização, amostras (em triplicata) com $30 \mathrm{~g}$ de solo úmido foram submetidas à extração e analisadas conforme descrito para o teste de recuperação do i.a.

Após a primeira semana, 3 mini-lisímetros sorteados foram desmontados e as colunas de solo divididas em camadas de $5 \mathrm{~cm}$. Cada camada foi homogeneizada, identificada, pesada e armazenada em congelador. Posteriormente, amostras em triplicata foram submetidas à extração conforme metodologia estabelecida.

A água percolada das colunas e recolhida nos garrafões de vidro foi medida e armazenada em local apropriado com temperatura controlada. Posteriormente, alíquotas dessa água foram submetidas à extração. As amostras de água de cada garrafão foram analisadas em triplicata observando-se o volume presente. Se o volume de água recolhido fosse superior a $40 \mathrm{~mL}$, cada amostra deveria ser de $10 \mathrm{~mL}$, e se o volume de água recolhido fosse inferior a $30 \mathrm{~mL}$, cada amostra deveria ter cerca de $30 \%$ do volume total. As amostras de água receberam 5 vezes o seu volume de diclorometano, sendo submetidas à extração conforme metodologia descrita para o teste de recuperação do i.a. a partir da água. Os extratos do solo e da água foram analisados por CLAE. Esses procedimentos foram repetidos semanalmente durante 10 semanas e a presença de i.a. em cada camada apresentada como porcentagem do inicialmente aplicado.

\section{RESULTADOS}

Os limites de quantificação e detecção do método foram estabelecidos estatisticamente por análise de regressão, com limite de confiança 95\%. O tempo de retenção do cumatetralila foi de 8,0 minutos nas condições apresentadas. A curva de calibração obtida a partir das soluçõespadrão testadas apresentou linearidade na faixa de concentração de 1,25 a $10 \mathrm{\mu g} \mathrm{mL}^{-1}$, coeficiente de correlação $(r)$ de 0,9999 e coeficiente de determinação $\left(r^{2}\right)$ de 0,9999 (Figura 1). Os limites de detecção e de quantificação para o i.a.cumatetralila foram de 0,2858 e $0,5610 \mu \mathrm{g}$ i.a. $\mathrm{mL}^{-1}$, respectivamente. 
FIGURA 1 - CROMATOGRAMAS DE CUMETATRALILA COM 10,0 $\mu \mathrm{g}(\mathrm{A})$; 5,0 (B); 2,5 (C) E 1,25 $\mu \mathrm{g}$ (D) DO INGREDIENTE ATIVO $\mathrm{mL}^{-1}$ DE SOLVENTE*

(a)

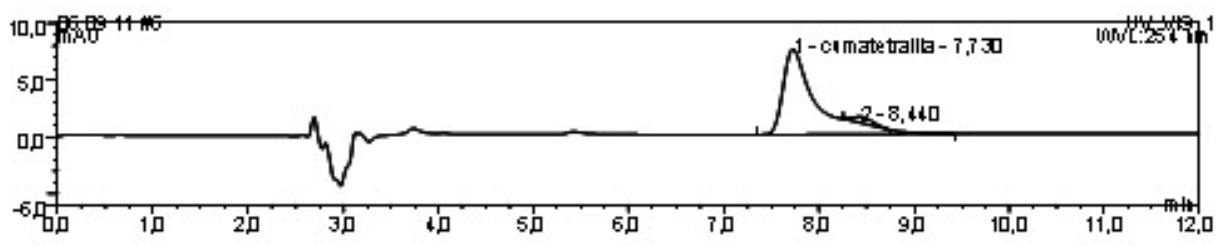

(b)

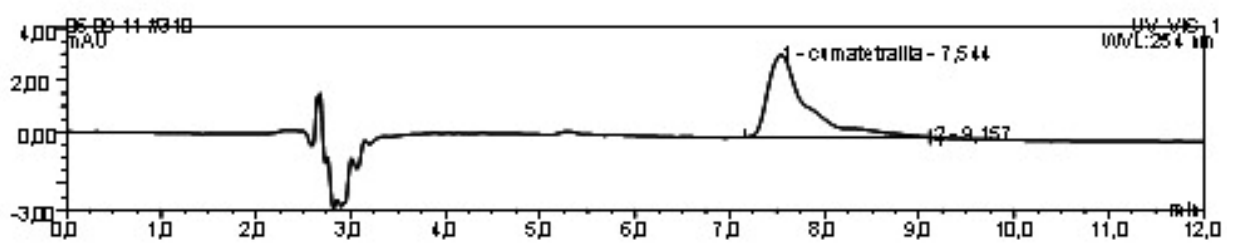

(c)

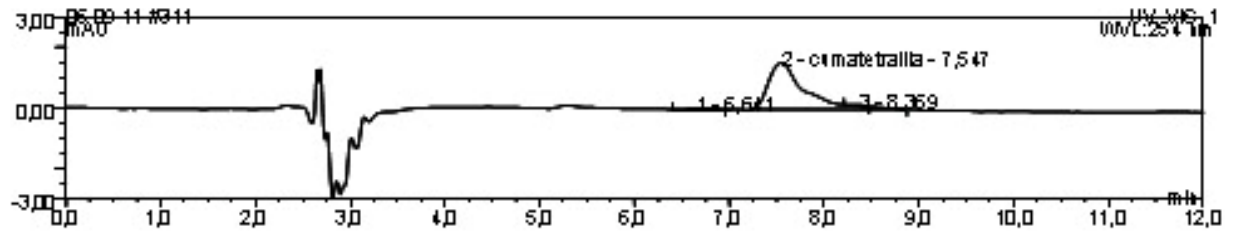

(d)

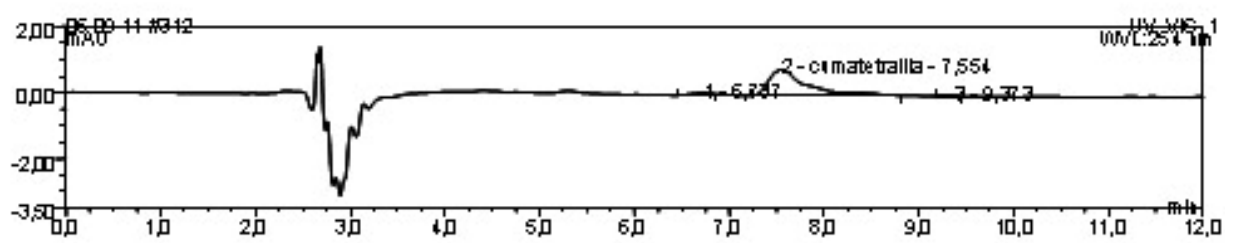

* Os cromatogramas mostram que até $1,25 \mu \mathrm{g}$ do ingrediente ativo $\mathrm{mL}^{-1}$ de solvente é possível detectar e quantificar a concentração presente na solução.

O solo utilizado nos ensaios é do tipo argiloso conforme determinado a partir das análises físico-químicas realizadas (Tabela 1).

\section{TABELA 1 - RESULTADOS DAS ANÁLISES FÍSICO-QUÍMICAS A PARTIR DE AMOSTRAS DO SOLO UTILIZADO NOS ENSAIOS COM O RATICIDA CUMATETRALILA}

\subsection{ESTUDO DE DISSIPAÇÃO E POTENCIAL DE LIXIVIAÇÃO - CUMATETRALILA}

Os testes de recuperação do i.a. de amostras de solo e de água tratadas (Figura 2) mostraram recuperação de $78,84 \% \pm 1,38$ e de $89,81 \% \pm 9,19$, respectivamente, sendo considerados satisfatórios. 


\section{FIGURA 2 - CROMATOGRAMAS DE AMOSTRAS DE SOLO (a) E DE ÁGUA (b) DE TESTES DE RECUPERAÇÃO DO INGREDIENTE ATIVO CUMATETRALILA E (c) DE SOLO BRANCO}

(a)

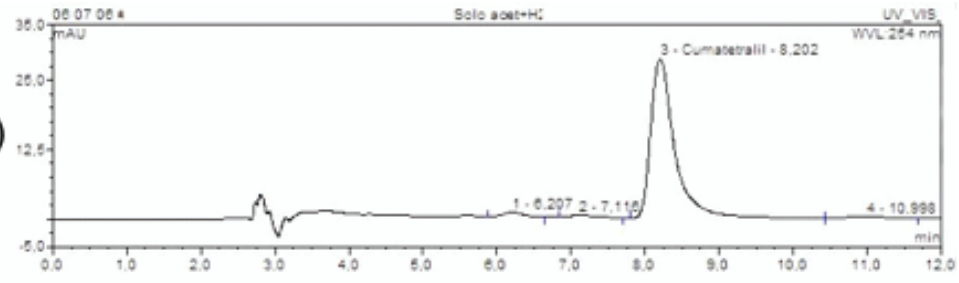

(b)

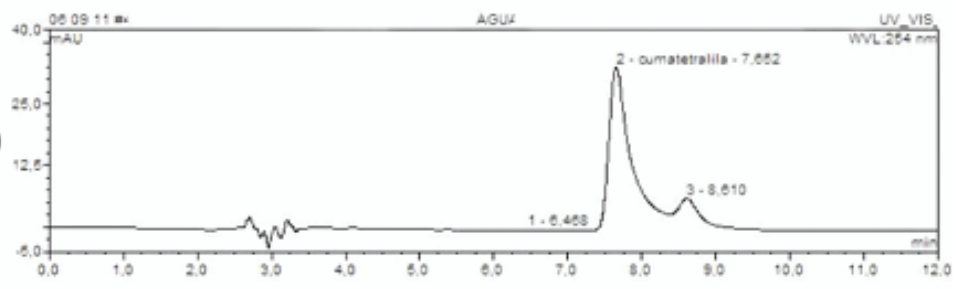

(c)

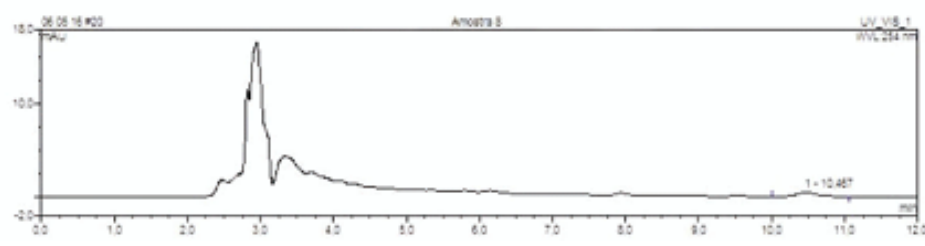

O resultados das extrações do solo no $\mathrm{T}_{\text {zero }}$ mostraram recuperação do i.a. em torno de $95 \%$ $(94,17 \% \pm 9,77$ - CV\% = 10,37\%), sendo, portanto, utilizada a mesma metodologia para análise das camadas de solo ao longo do experimento. A análise dos extratos obtidos a partir de amostras das camadas de solo durante as 10 semanas de estudo são mostrados na Tabela 2. As camadas inferiores foram analisadas somente quando se detectou a presença de i.a. na camada imediatamente superior.

\section{TABELA 2 - RECUPERAÇÃO DO INGREDIENTE ATIVO CUMATETRALILA DAS CAMADAS DE SOLO TRATADO COM O RATICIDA DURANTE 10 SEMANAS*}

\begin{tabular}{lcccc}
\hline SEMANA & \multicolumn{4}{c}{ CAMADA DE SOLO } \\
& $0-5 \mathrm{~cm}$ & $5-10 \mathrm{~cm}$ & $10-15 \mathrm{~cm}$ & $15-20 \mathrm{~cm}$ \\
\hline $\mathrm{T}_{1}$ & $77,06 \pm 7,19$ & $\mathrm{NQ}$ & $\mathrm{ND}$ & $\mathrm{NR}$ \\
$\mathrm{T}_{2}$ & $74,34 \pm 2,27$ & $\mathrm{NQ}$ & $\mathrm{ND}$ & $\mathrm{NR}$ \\
$\mathrm{T}_{3}$ & $60,03 \pm 3,23$ & $\mathrm{NQ}$ & $\mathrm{ND}$ & $\mathrm{NR}$ \\
$\mathrm{T}_{4}$ & $53,20 \pm 5,93$ & $\mathrm{NQ}$ & $\mathrm{ND}$ & $\mathrm{NR}$ \\
$\mathrm{T}_{5}$ & $25,55 \pm 5,24$ & $4,54 \pm 0,94$ & $\mathrm{ND}$ & $\mathrm{NR}$ \\
$\mathrm{T}_{6}$ & $19,91 \pm 5,02$ & $6,51 \pm 0,82$ & $\mathrm{ND}$ & $\mathrm{NR}$ \\
$\mathrm{T}_{7}$ & $22,02 \pm 4,39$ & $\mathrm{NQ}$ & $\mathrm{ND}$ & $\mathrm{NR}$ \\
$\mathrm{T}_{8}$ & $22,76 \pm 1,82$ & $\mathrm{NQ}$ & $\mathrm{ND}$ & $\mathrm{NR}$ \\
$\mathrm{T}_{9}$ & $11,74 \pm 2,09$ & $\mathrm{NQ}$ & $\mathrm{ND}$ & $\mathrm{NR}$ \\
$\mathrm{T}_{10}$ & $7,33 \pm 0,58$ & $4,56 \pm 0,37$ & $\mathrm{ND}$ & $\mathrm{NR}$ \\
\hline
\end{tabular}

* Resultados em porcentagem (média \pm desvio-padrão) de recuperação do ingrediente ativo inicialmente aplicado sobre o solo.ND = não-detectado. NQ = detectado, mas não quantificado. NR = não-realizada. 
A Figura 3 mostra cromatogramas de amostras de solo tratado com o pó de contato, na $5^{\mathrm{a}} \mathrm{e}$ na $10^{\mathrm{a}}$ semana ( $\mathrm{T}_{5} \mathrm{e} \mathrm{T}_{10}$, respectivamente), nas camadas de 0 a $5 \mathrm{~cm}$ (superficial), 5 a $10 \mathrm{~cm}$ e 10 a 20 $\mathrm{cm}$ de profundidade, bem como amostra de água coletada até a $5^{\mathrm{a}}$ semana.

\section{FIGURA 3 - CROMATOGRAMAS DE AMOSTRAS DE SOLO TRATADO COM CUMATETRALILA EXPOSTO AO AMBIENTE POR 5 SEMANAS $(a, b, c)$, DA ÁGUA COLETADA NESSE PERÍODO (d) E POR 10 SEMANAS (e)}

(a)

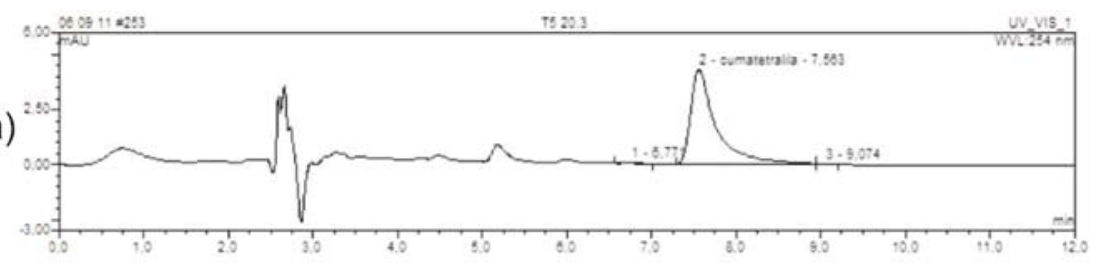

(b)

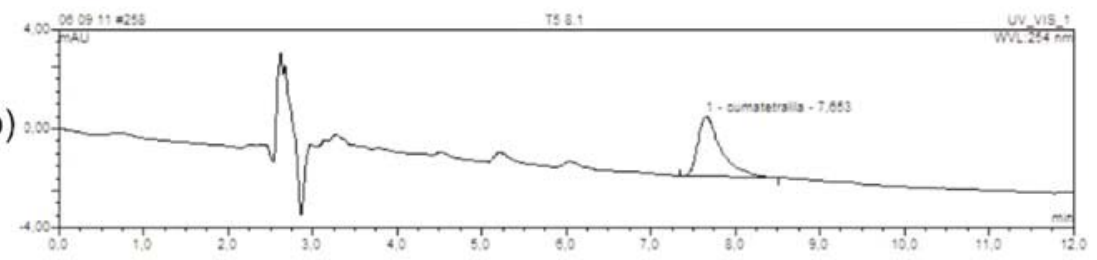

(c)

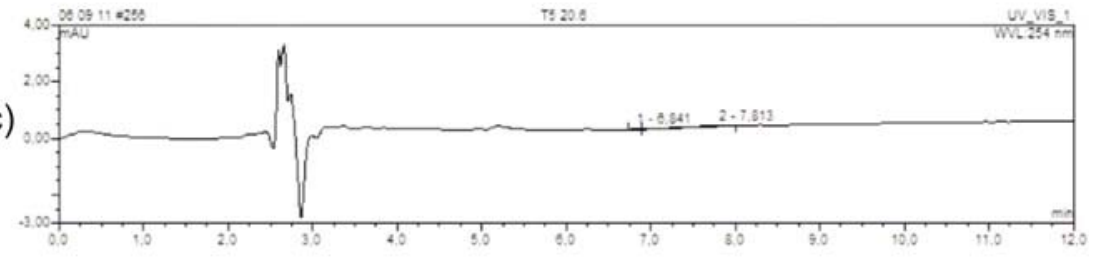

(d)

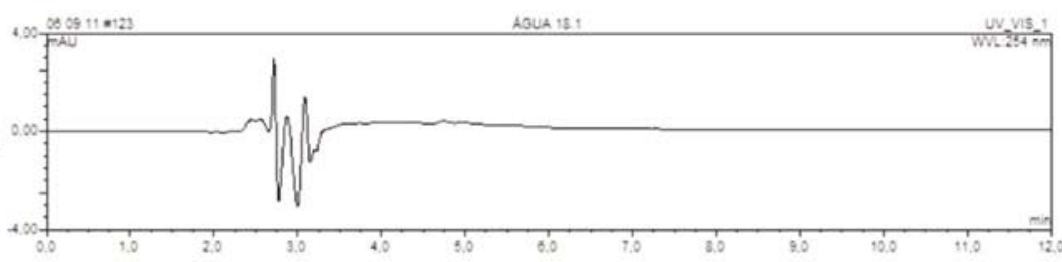

(e)

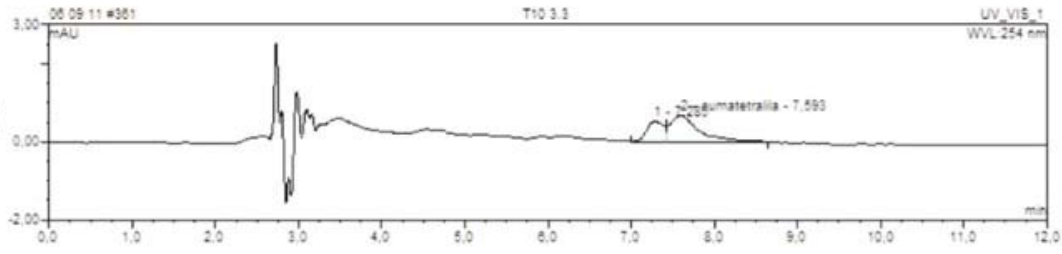

Em (a) e (e) amostras de solo da camada de 0 a $5 \mathrm{~cm}$, em (b) de 5 a $10 \mathrm{~cm}$ e em (c) 10 a $20 \mathrm{~cm}$ de profundidade.

A Figura 4 mostra a diminuição na recuperação do i.a. durante as 10 semanas de estudo $\left(T_{1}, T_{2}, T_{3}, T_{4}, T_{5}, T_{6}, T_{7}, T_{8}, T_{9}\right.$ e $\left.T_{10}\right)$. Não se detectou a presença do i.a. em nenhuma das análises realizadas nas amostras de água coletada de cada mini-lisímetro durante as 10 semanas. 


\section{FIGURA 4 - DIMINUIÇÃO DA CONCENTRAÇÃO DO INGREDIENTE ATIVO CUMATETRALILA ( $\mu$ g i.a. $g^{-1} S O L O$ ) PRESENTE NAS AMOSTRAS DE SOLO DA CAMADA $0-5 \mathrm{~cm}$ ANALISADAS DURANTE AS 10 SEMANAS DE ESTUDO}

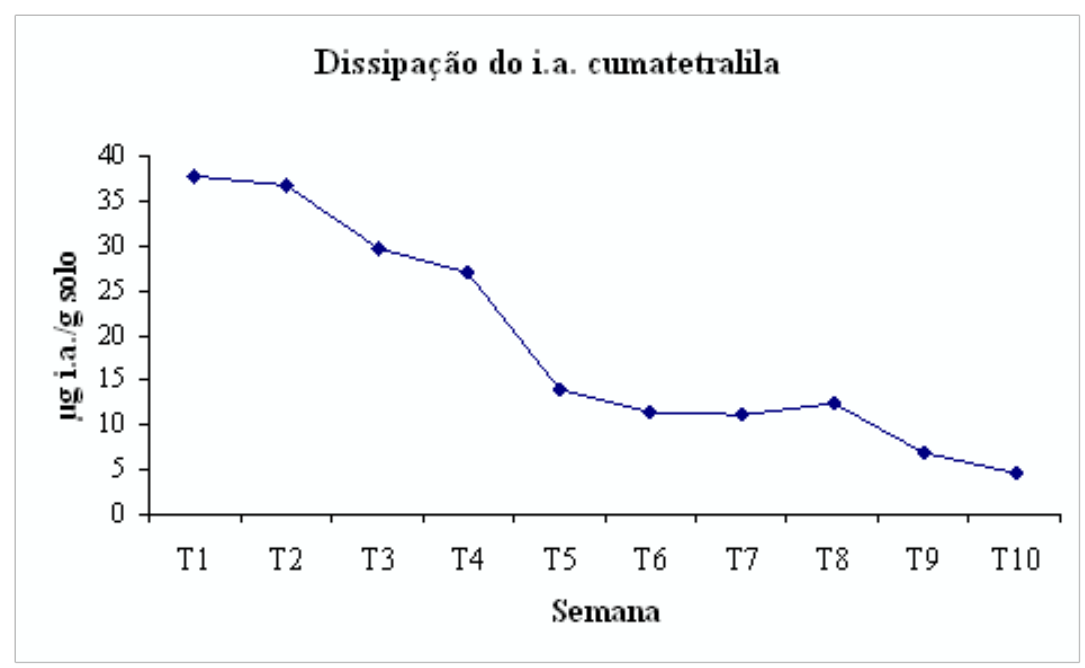

$\mathrm{O} \mathrm{T}_{\text {zero }}$ com 56,82 $\mu$ g i.a. $\mathrm{g}^{-1}$ solo, o que corresponde a cerca de 95\% de recuperação do aplicado, foi utilizado como parâmetro para comparação com as 10 (dez) semanas de estudo.

A Tabela 3 mostra as variações de pluviosidade e as temperaturas mínima e máxima durante as 10 semanas de estudo.

TABELA 3 - CONDIÇÕES CLIMÁTICAS, PLUVIOSIDADE, TEMPERATURA MÍNIMA E
TEMPERATURA MÁXIMA, MEDIDAS SEMANALMENTE DURANTE 10 SEMANAS

\begin{tabular}{cccccccccccc}
\hline \multicolumn{2}{c}{ SEMANAS } & T1 & T2 & T3 & T4 & T5 & T6 & T7 & T8 & T9 & T10 \\
\hline \multicolumn{2}{c}{ Pluviosidade $(\mathrm{mm})$} & 70 & 3 & 1 & 23 & 154 & 103 & 0 & 42 & 55 & 98 \\
Temp & MIN & 15 & 12 & 13 & 15 & 19 & 17 & 17 & 19 & 19 & 19 \\
$\left({ }^{\circ} \mathrm{C}\right)$ & MAX & 34 & 24 & 30 & 34 & 30 & 25 & 26 & 34 & 35 & 21 \\
\hline
\end{tabular}

\section{DISCUSSÃO}

Foram realizadas extrações de amostras de solo das camadas de $0-5 \mathrm{~cm}$ e $5-10 \mathrm{~cm}$ nas 10 semanas $\left(\mathrm{T}_{1} \mathrm{a} \mathrm{T}_{10}\right)$. Observou-se diminuição na concentração do i.a. por grama de solo ao longo das 10 semanas de estudo nas amostras da camada de solo $0-5 \mathrm{~cm}$ de cerca de $37 \mu \mathrm{g}$ i.a. $\mathrm{g}^{-1}$ solo na primeira semana para $4,58 \mu \mathrm{g}$ i.a. $\mathrm{g}^{-1}$ solo na décima semana (cerca de $84 \%$ ). Embora tenha ocorrido diminuição da concentração do i.a., após 10 semanas ainda foi possível detectar e quantificar o produto no ambiente. Tal fato merece alerta devido a aplicação desse tipo de raticida ser periódica e em locais com condições ambientais e sanitárias precárias (margens de córregos, muitas vezes ocupadas por favelas com vielas, piso das casas sem pavimentação, e uso de água de solução alternativa, inclusive poços rasos). O contato prolongado, em especial, de crianças com o solo pode acarretar risco de contaminação. Muitos agrotóxicos podem ser absorvidos pelas raízes e translocados para todos os tecidos vegetais, acumulando-se em diferentes órgãos e tecidos, sendo que a concentração do produto está geralmente associada ao tempo de exposição ao mesmo (DOSHI e THAKORE, 1995). Não se encontrou referências da possibilidade de ocorrência desse fenômeno com o raticida 
cumatetralila. Além disso, é importante salientar que o uso de substâncias químicas constitui risco potencial de contaminação ambiental e bioacumulação. Os organismos que vivem no solo ou sedimentos são diretamente expostos e podem bioacumular tais compostos em seus tecidos (AMORIN et al., 2002).

Na primeira $\left(T_{1}\right)$ e na segunda semana $\left(T_{2}\right)$, cerca de $75 \%$ do i.a. inicialmente aplicado foi recuperado da camada de $0-5 \mathrm{~cm}$. Portanto, $25 \%$ foi dissipado a partir da superfície do solo que recebeu a aplicação. Como o cumatetralila é rapidamente degrado sob ação da luz solar ou UV (WORTHING e HANCE, 1991), sua exposição direta à luz pode ter favorecido a dissipação da camada superficial do solo a partir da primeira semana após a aplicação. Na análise das amostras de solo da camada $5-10 \mathrm{~cm}$ nas 2 primeiras semanas, o i.a. foi detectado mas não quantificado por se apresentar abaixo do limite de quantificação do método (2,5 $\mu$ g i.a. $\mathrm{g}^{-1}$ solo).

É interessante observar que após a semana $\mathrm{T}_{3}$, embora já não se notasse a presença do raticida sobre o solo, detectou-se e quantificou-se o i.a. nas amostras analisadas a partir da camada $0-5 \mathrm{~cm}$ do solo. Isso evidencia que o fato de não se observar o pó de contato sobre o solo não garante a ausência do i.a. no ambiente. Essa constatação é importante, pois muitas vezes, as pessoas não vendo nada sobre o solo acabam manipulando-o diretamente e correndo o risco de exposição ao composto. Outra questão refere-se ao potencial de atuação no organismo-alvo, no caso os roedores, ao transitar sobre a camada de solo contaminada.

As porcentagens de recuperação do i.a. na primeira camada de solo $(0-5 \mathrm{~cm})$ nas semanas $\mathrm{T}_{3}$ e $\mathrm{T}_{4}$ foram de 60 e $53 \%$ respectivamente. $\mathrm{O}$ i.a. foi detectado, mas não quantificado na segunda camada $(5-10 \mathrm{~cm})$, mostrando que até 40 e $47 \%$ do i.a. na semana $\mathrm{T}_{3}$ ena semana $\mathrm{T}_{4}$, respectivamente, foram dissipados a partir da superfície do solo em que foi aplicado, mas encontrando-se presente na camada inferior.

Na semana $\mathrm{T}_{5}$, cerca de $25 \%$ do i.a. foi recuperado a partir de amostras da camada de solo de $0-5 \mathrm{~cm}$ e aproximadamente $4 \%$ na camada seguinte (Tabela 2). Na semana $\mathrm{T}_{6}$, cerca de $20 \%$ do i.a. foi recuperado a partir de amostras da camada de solo de $0-5 \mathrm{~cm}$ e $6 \%$ aproximadamente na camada seguinte (Tabela 2). Como na quinta e na sexta semanas a pluviosidade foi acentuada, superando $100 \mathrm{~mm}$, pode ter sido favorecido o arraste do produto à camada de $5-10 \mathrm{~cm}$ (Figura 5). Esses resultados preliminares mostraram que algumas semanas após a aplicação sobre a superfície do solo, o i.a. pode ser lixiviado à segunda camada, especialmente sob fortes chuvas.

\section{FIGURA 5 - VARIAÇÃO NA PLUVIOSIDADE AO LONGO DAS 10 (DEZ) SEMANAS DE ESTUDO}

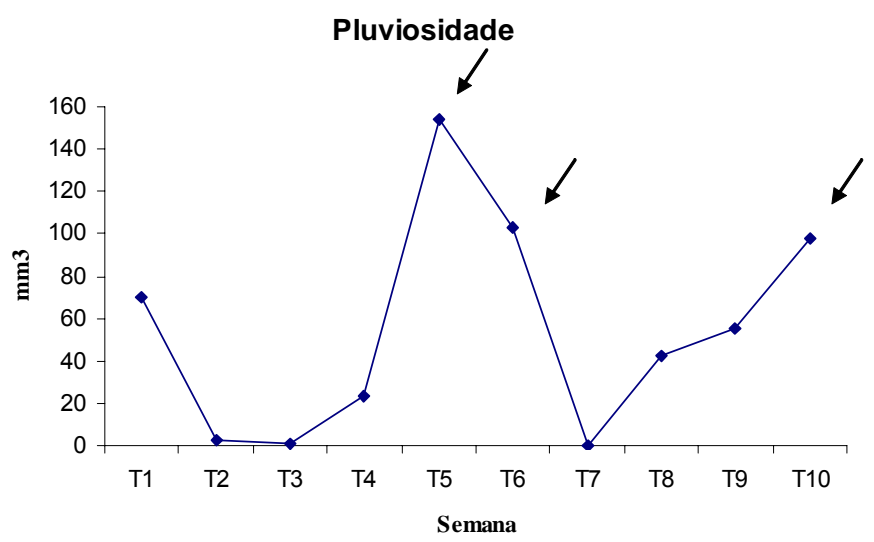

As setas indicam as semanas $\left(t_{5}, t_{6}\right.$ e $\left.t_{10}\right)$ nas quais o ingrediente ativo cumatetralila foi detectado e quantificado na camada de $0-5 \mathrm{~cm}$. 
Detectou-se a presença do i.a nas semanas $T_{7}, T_{8}, T_{9}$, mas não foi possível sua quantificação em razão do limite de quantificação do método de análise. Embora não tenha sido quantificado o i.a. presente na segunda camada de solo $(5-10 \mathrm{~cm})$, sua detecção causa certa apreensão devido ao produto ser aplicado periodicamente na entrada das tocas. Na semana $T_{10}$, cerca de $7,0 \%$ do i.a. foi detectado na camada $0-5 \mathrm{~cm}$ e $4,0 \%$ na camada de $5-10 \mathrm{~cm}$, semelhante ao que ocorreu na quinta e na sexta semanas. Também nesse período houve pluviosidade significativa $(98 \mathrm{~mm})$, o que pode ter favorecido o arraste do produto à camada de $5-10 \mathrm{~cm}$ (Figura 2).

Nas amostras de solos da camada $10-15 \mathrm{~cm}$ analisadas não foi detectada a presença do i.a. cumatetralila durante as 10 semanas de estudo e, portanto, não foram realizadas análises de amostras de solo da camada $15-20 \mathrm{~cm}$. Isso indica que o composto fica retido nas camadas iniciais do solo, podendo ser degradado física, química ou biologicamente.

Os resultados mostraram que mesmo após 10 semanas de aplicação do raticida formulado como pó de contato sobre a superfície do solo, cerca de 7,0\% do i.a. ainda se encontrava na camada superficial do solo. Assim, aplicações periódicas e sucessivas no mesmo local devem ser precedidas de cuidados.

Não se detectou a presença do i.a. cumatetralila nas amostras de água das 10 semanas analisadas, indicando que o mesmo ficou retido nas camadas de solo superficiais. Os resultados apontaram que no tipo de solo usado não ocorreu lixiviação do i.a. para suas camadas mais profundas, revelando baixo risco de contaminação da água subterrânea. Embora o cumatetralila não seja fortemente adsorvido ao carbono orgânico e à argila do solo (TOXNET, 2004), o fato de ter se utilizado solo argiloso pode ter dificultado a lixiviação do produto devido ao menor espaço disponível para o movimento de partículas. Nos solos de predominância argilosa há menor aeração e maior retenção de água e de outras moléculas. Já nos solos arenosos ocorre o inverso pela tendência de maior aeração e transporte de água e nutrientes em razão do maior espaçamento entre as partículas predominantes (PRADO, 2003). Assim, os resultados poderiam ser diferentes em solo arenoso com mais espaço entre as partículas permitindo maior mobilidade da água, ar e compostos presentes.

\section{CONCLUSÃO}

Ocorreu diminuição da concentração do ingrediente ativo cumatetralila na camada superficial do solo ao longo das 10 semanas de estudo, embora o produto ainda tenha sido detectado na décima semana. Baixas concentrações do ingrediente ativo cumatetralila chegaram a $10 \mathrm{~cm}$ do perfil do solo, mas não atingiram camadas mais profundas. Não se detectou o ingrediente ativo cumatetralila nas amostras de água analisadas nas 10 semanas de estudo. Os resultados obtidos permitem usar o raticida com maior segurança, mas há necessidade de se verificar a ocorrência de lixiviação aplicandose o produto em outros tipos de solo, principalmente em solos arenosos, nos quais há maior possibilidade de movimentação de partículas.

\section{ABSTRACT}

\section{ENVIRONMENTAL BEHAVIOUR OF COUMATETRALYL RODENTICIDE IN CONTACT POWDER}

In the chemical control of rodents in the São Paulo city (Brazil), coumatetralyl contact powder is applied in the entrance of the burrows, being subject to the action of bad weather, that could imply in alterations in the active ingredient (a.i.) and transference to other environments. In order to verify these possibilities, the rodenticide was applied in experimental systems simulating the field conditions. Soil and water samples were collected weekly, for 10 weeks, and analyzed by high performance liquid chromatography. The obtained data were used for the evaluation of the dissipation of a.i. and of the lixiviation potential in the profile of the soil. The results pointed coumatetralyl presence in the layer 0 to $5 \mathrm{~cm}$ of the soil during the 10 weeks. It was detected about $75 \%$ of a.i. applied in the first 3 weeks, about $20 \%$ between $5^{\text {th }}$ and 
$8^{\text {th }}$ weeks and about $7 \%$ in the $10^{\text {th }}$ week. In water samples of a.i it was not detected, during the 10 weeks. It was concluded that the a.i. stays in the environment for at least 10 weeks, but it doesn't reach the underground water in the type of used soil.

KEY-WORDS: HYDROXYCOUMARIN; LIXIVIATION; PERSISTENCE; RODENTICIDE.

\section{REFERÊNCIAS}

1 ALMEIDA, L.P.; MARTINS, L.F.S.; BROD, C.S.; GERMANO, P.M.L. Levantamento soroepidemiológico de leptospirose em trabalhadores do serviço de saneamento ambiental em localidade urbana da região sul do Brasil. Revista de Saúde Pública, v. 28, p.1-9, 1994.

2 AMORIM, M.J.B.; SOUSA, J.P.; NOGUEIRA, J.Á.; SOARES, A.M.V.M. Bioaccumulation and elimination of ${ }^{14} \mathrm{C}$-lindane by Enchytraeus albidus in artificial (OECD) and a natural soil. Chemosphere, v. 49, p.323$329,2002$.

3 BRASIL. Ministério da Saúde. Manual de controle de roedores. Brasília: Fundação Nacional da Saúde, 2002. $132 \mathrm{p}$.

4 COMER, J.A.; PADDOCK, C.D., CHILDS, J.E. Urban zoonosis caused by Bartonella, Coxiella, Ehrlichia and Rickettsia species. Vector Borne Zoonotic Diseases, v. 1, p. 91-118, 2001.

5 DOSHI, A.; THAKORE, B.B.L. Uptake translocations and residues of metalaxyl in opium plants. Indian Journal Plant Protection, v. 23, p. 191-194, 1995.

6 EMBRAPA. Empresa Brasileira de Pesquisa Agropecuária. Determinação da umidade, pH e capacidade de retenção de água do solo. In: INDICADORES biológicos e bioquímicos da qualidade do solo. Jaguariúna, 2000. p. 37-40. (Manual Técnico).

7 EPA. Environmental Protection Agency. Registration Eligibility Decision (RED). July, 1998. Disponível em: http://www.epa.gov/REDs/2100red.pdf. Acesso em: 25/mar/2005.

8 KOOKANA, R.S.; DI, H.J.; AYLMORE, L.A.G. A field study of leaching and degradation of nine pesticides in a sandy soil. Australian Journal Soil Research, v. 33, p. 1019-1030, 1996.

9 LARINI L. Toxicologia dos praguicidas. São Paulo: Manole,1999.

10 MEIER, P.C.; ZÜND R.E. Statistical methods in analytical chemistry. New York: John Wiley \& Sons, 1993.

11 MORIN, M.F.; MERLET, N.; DORE M.; LECHEVIN J.C. Analyse de résidus de bromadiolone (rodenticide anticoagulant) dans l'eau et les tissus animaux. Analysis, v. 7, p. 526-531, 1989.

12 PAPINI, S.; HOMEM DE MELO, M.H.S.; OLIVEIRA, D.C.; ANDRÉA, M.M.; DAL BOM, M.G.; CREOLEZ, E.F.A; LUCHINI, L.C. O uso de inseticidas e raticidas no controle da fauna sinantrópica no município de São Paulo: contaminação da população e do ambiente? Revista de Vigilância Sanitária, v. 1, p. 174-179, 2005.

13 PETROVIC, A.M.; BARRET W.C.; LARSSON-KOVACH, I.M.; REID, C.M.; LISK, D.J. The influence of peat amendment and turf density of downward migration of metalaxyl fungicide in creeping bentgrass sand lysimeters. Chemosphere, v. 3, p. 2335-2340, 1997.

14 PRADO L. Solos do Brasil. 3. ed. Piracicaba: ESALQ/USP, 2003. 275 p.

15 RACKE, K.D. Pesticides in the soil microbial ecosystem. In: RACKE, K.D.; COATS, J.R. (Org).. Enhanced biodegradation of pesticides in the environment. Washington: American Chemical Society, 1990. p.1-12.

16 RAO, S.R.; GUPTA, N.; BHALLA, P.; AGARWAL, S.K. Leptospirosis in Indian and the rest of the world. Brazilian Journal of Infectious Diseases, v. 3, p. 178-193, 2003.

17 SEMA. Secretaria Especial do Meio Ambiente do Paraná. Manual de avaliação de impactos ambientais. 2. ed Curitiba, 1993. 
18 SILVA H.R.; TAVARES-NETO, J.; BINA, J.C.; MEYER, R. Leptospiral infection and subclinical presentation among children in Salvador, Bahia. Revista da Sociedade Brasileira de Medicina Tropical, v. 36, p. 227233, 2003.

19 TOXNET. Toxicology Data Network. United States National Library of Medicine. Coumatetralyl. Disponível em: http://toxnet.nlm.nih.gov/cgi-bin/sis/search. Acesso em: 29/mar/2004.

20 VIEIRA, J.B.; ALMEIDA, A.M.P.; ALMEIDA, C.R. Epidemiologia e controle da peste no Brasil. Revista da Sociedade Brasileira de Medicina Tropical. v. 27, p. 51-58, 1994.

21 WORTHING, C.R.; HANCE, J. (ed.) The pesticide manual - World compendium. 9th ed. Surrey, UK: The British Crop Protection Council, 1991. p.188-189. 